\title{
APLICAÇÃO DE CALCÁRIO E FÓSFORO E ESTABILIDADE DA ESTRUTURA DE UM SOLO ÁCIDO(1)
}

\author{
J . A. AlbUQUERQUE ${ }^{(2)}$, C. BAYE ${ }^{(3,5)}$, P. R. ER NANI ${ }^{(2,5)}$, \\ A. L. MAFRA ${ }^{(2)} \&$ E. C. FONTANA ${ }^{(4)}$
}

\begin{abstract}
RESUMO
O presente estudo objetivou avaliar o efei to da aplicação de calcário e fósforo sobre o comportamento estrutural de um solo ácido com altos teores de argila e matéria orgânica, típico do planalto sul brasileiro. 0 experimento foi realizado no campo, num Latossolo Bruno, em Lages (SC), no delineamento de blocos ao acaso, disposto em parcelas subdivididas. Os tratamentos consistiram da aplicação, no início do experi mento, de 0, 4,5 e 9,0 $\mathrm{Mg} \mathrm{ha}^{-1}$ de calcário, combi nada com adições anuais médias de fósforo correspondentes a 42, 84, 126 e $168 \mathrm{~kg} \mathrm{ha}^{-1}$ de $\mathrm{P}_{2} \mathrm{O}_{5}$. Determinaram-se o grau de floculação da argila, a estabilidade de agregados, a condutividade elétrica, o ponto de efeito salino nulo (PESN) e a composição química do solo. A dispersão da argila aumentou linearmente com a dose de calcário aplicada, o que foi relacionado com a elevação do PESN e do potencial elétrico superficial negativo. Essas alterações nos atributos fisicoquímicos dos colói des de solo não interferiram, entretanto, sobre a estabilidade estrutural avaliada pelo diâmetro médio ponderado dos agregados, o que pode estar relacionado com o efeito indireto da calagem sobre a agregação graças ao maior aporte de resíduos vegetais ao solo e do estímulo à atividade biológica do solo, além da própria adição de $\mathrm{Ca}$. A adição de $\mathrm{P}$ não alterou nenhum atri buto físico analisado. A adição de calcário, apesar de aumentar a dispersão da argila, não comprometeu a qualidade física desse solo argil loso com alto tamponamento.
\end{abstract}

Termos de indexação: calagem, agregação, dispersão, PESN, solos de carga variável.

\footnotetext{
(1) Trabal ho apresentado na $23^{a}$ Reunião Brasileira de Manejo e Conservação do Solo e Água, I lhéus, 2000. Recebido para publicação em outubro de 2002 e aprovado em maio de 2003.

(2) Professor do Departamento de Solos, Universidade do Estado de Santa Catarina - UDESC. Caixa Postal 281, CEP 88520-000 Lages (SC). E-mail: a2ja@cav.udesc.br

(3) Professor do Departamento de Solos, Universidade Federal do Rio Grande do Sul - UFRGR. Agronomia, Caixa Postal 776, CEP 90001-970 Porto Alegre (RS). E-mail: cimelio.bayer@ufrgs.br

(4) Engenheiro-Agrônomo, UDESC.

(5) Bolsista do CNPq.
} 


\title{
SUMMARY: EFFECTS OF LIMING AND PHOSPHORUS APPLICATION ON THE STRUCTURAL STABILITY OF AN ACID SOIL
}

\begin{abstract}
Theobjective of this field experiment was to evaluatetheeffect of liming and phosphorus application on thestructural stabi lity of a clayey acid soil with high organic matter content, a typical feature of the southern Brazilian plateau. The study was carried out on a Brown Latosol (Haplohumox), in Lages, State of Santa Catarina, Brazil, in a split plot arrangement of a completely randomized block design. Treatments consisted of limeapplication at rates of $0,4.5$, and $9.0 \mathrm{Mg} \mathrm{ha}^{-1}$ at the beginning of the experiment, combined with mean annual $\mathrm{P}$ applications of $42,84,126$, and $168 \mathrm{~kg} \mathrm{ha}^{-1}$ of $\mathrm{P}_{2} \mathrm{O}_{5}$. Theclay flocculation degree, aggregate stability, electrical conductivity, point of zero salt effect (PZSE), and the chemical soil composition were determined. Clay dispersion increased linearly with theliming rate, and was related to theincreasein PZSE and negativesurface ed ectric potential. Thesealterations in the physicochemical attributes of the soil colloids, however, had no influence on the structural stability of the soil, as evidenced by themean weight diameter of theaggregates. This might bea consequence of the indirect positive effects of liming on soil aggregation as a consequence of theincreased biological soil activity, as wel as the beneficial influence of $\mathrm{Ca}$ addition. Phosphorus fertilizers did not affect any soil physical attributes. Liming, in spite of increasing clay dispersion, had no detrimental affect on the physical quality of this highly buffered soil.
\end{abstract}

Index terms: lime, clay dispersion, PZSE, soil aggregation, variablecharge soils.

\section{NTRODUÇÃO}

A calagem e a adubação fosfatada são práticas de manejo da fertilidade do solo que el evam o rendimento das culturas, especialmente nas regiões demaior altitude do sul do Brasil, onde predominam sol os extremamente áci dos e deficientes em fósforo (Ernani et al., 2000). A aplicação de cal cário eleva os teores de Ca e Mg, diminui ou elimina oAl trocável e aumenta as cargas negativas nesses solos que têm predomínio de cargas variáveis. Essas alterações químicas podem, entretanto, influenciar alguns atributos físicos do solo, por al terar ocomportamento el etroquímico dos colóides. Esses efeitos são complexos e muitas interações podem ocorrer por meio de mecanismos muitas vezes ainda pouco conhecidos (Haynes \& N aidu, 1998).

As mudanças na estrutura do sol o eo rompimento dos agregados decorrentes do uso agrícola indevido normalmente acarretam restrições ao crescimento radicular, reduzindo a exploração de água e nutrientes pelas culturas. Essas alterações são, muitas vezes, manifestadas pela compactação acentuada, diminuindo a porosidade e a infiltração de água no solo, aumentando o acúmulo de água sobre a superfície e a erosão hídrica. O selamento superficial do solo nessas condições restringiria a emergência das plantas e poderia favorecer a ocorrência de doenças radiculares. Em conjunto, essas modificações físicas viriam a comprometer o desenvolvimento das culturas aí presentes.

As alterações nos atributos físicos de solos com colóides de carga variável, como os Latossolos, em especial na camada superficial mais rica em matéria orgânica, podem ser provocadas por diversos mecanismos, a saber: (a) quebra dos agregados por efeito mecânico causado pelo revolvimento do solo ou pisoteio animal (Fontes et al., 1995); (b) perda da matéria orgânica, que é um dos principais agentes cimentantes das partículas do solo, e (c) alterações químicas advindas do uso de corretivos e adubos (Westerhof et al., 1999).

As mudanças na estabilidade de agregados e outros atributos físicos do solo, decorrentes da calagem eda adubação, podem estar ligadas a dois mecanismos principais. O primeiro deles refere-se ao efeito direto detais práticas, quemodificam a composição química da solução do solo. Assim, as alterações no pH, na força iônica da sol ução e nos tipos deíons presentes, influenciariam significativamente a dispersão ou floculação dos colóides, interferindo, por conseqüência, na agregação das partículas do solo (Seta \& Karathanasis, 1997). O segundo mecanismo referese ao efeito indireto da adição de cal cário e adubos ao sol o, que favoreceria a produção de fitomassa das culturas, levando a uma maior adição de matéria orgânica, que incrementaria a atividade mi crobiana e a estabilidade dos agregados (Haynes, 1984).

Algumas dessas alterações nos atributos físicos do solo advindas da adição de cal cário e fosfato são relatadas por Butierres (1980) e por Morelli \& Ferreira (1987), que estudaram, em laboratório, o efeito dessas práticas sobre o comportamento físicohídrico de solos da regiãosul do Brasil. Nesses casos, os efeitos variaram entre os horizontes e entre os solos eforam condicionados principalmente pelo teor 
de matéria orgânica do solo. Outro estudo desenvolvido em campo por Albuquerque et al. (2000), num Latossolo Bruno da região sul, evidenciou o papel isolado da calagem na alteração dos atributos físicos do solo.

O presente estudo objetivou avaliar o efeito da adição de cal cário e fósforo sobre o comportamento eletroquímico de superfície das partículas e sua relação com a estabilidade da estrutura de um solo altamente tamponado.

\section{MATERIAL E MÉTODOS}

No estudo, utilizaram-se amostras de solo, coletadas em setembro de 1999, em experimento realizado, durante cinco anos, na região do planalto catarinense, em Lages (SC). O solo é um Latossolo Bruno argiloso álico (Haplohumox), derivado de basalto, e a altitude do local é de $937 \mathrm{~m}$. A mineral ogia da fração argila é predominantemente caulinítica e em menor quantidade, argilominerais 2:1 com hidróxi-Al nas entrecamadas, gibbsita e goethita (Albuquerque et al., 2000). O clima da região é do tipo mesotérmi co úmi do com verão fresco, $\mathrm{Cfb}$, segundo a classificação de Köppen. A temperatura média anual é de 15,6 ㅇ $\mathrm{C}$ e a preci pitação média anual écerca de 1.400 mm (Santa Catarina, 1986).

Os tratamentos consistiram de três doses de calcário dolomítico $\left(0,4,5\right.$ e $\left.9,0 \mathrm{Mg} \mathrm{ha}^{-1}\right)$ correspondentes a $100 \%$ de poder relativo de neutralização. Os níveis de calagem foram combinados com doses crescentes de fósforo, a saber: $\mathrm{P}_{42}, \mathrm{P}_{84}, \mathrm{P}_{126} \mathrm{eP}_{168}$, correspondendo, respectivamente, a aplicações de $\mathrm{P}_{2} \mathrm{O}_{5}$, no primeiro ano, de 60,120 , 180 e $240 \mathrm{~kg} \mathrm{ha}^{-1}$; no segundo, quarto e quinto ano, de 40, 80, 120 e $160 \mathrm{~kg} \mathrm{ha}^{-1}$, e, no terceiro ano, de $30,60,90$ e $120 \mathrm{~kg} \mathrm{ha}^{-1}$, resultando numa dosemédia anual de 42, 84, 126 e $168 \mathrm{~kg} \mathrm{ha}^{-1} \mathrm{de}_{2} \mathrm{O}_{5}$. O fósforo foi adicionado na forma de superfosfato triplo. O delineamento experimental foi de blocos ao acaso, em parcel as subdi vidi das, com quatro repetições. 0 cal cário e o adubo fosfatado foram distribuídos uniformemente a lanço e incorporados ao solo até aproximadamente $17 \mathrm{~cm}$ de profundidade, com duas arações e duas gradagens. O plantio de milho foi feito no verão, após pousio de inverno, com uso do preparo convencional do solo nos primeiros quatro anos e semeadura direta no ano anterior à amostragem do sol o utilizada neste estudo.

As amostras de solo foram coletadas na camada de $0-10 \mathrm{~cm}$ de profundidade, com duas subamostras por tratamento, encami nhadas aolaboratório e secas em temperatura ambiente. Na terra fina seca ao ar, foram analisadas a condutividade elétrica em extrato solo-água destilada de 1:5 em volume (Tedesco et al., 1995) e a argila dispersa em água e em $\mathrm{NaOH} 1 \mathrm{~mol} \mathrm{~L}^{-1}$, pelo método da pipeta, com posterior cálculo do grau de floculação da argila (EMBRAPA, 1997). A estabilidade da estrutura do sol o foi avaliada pel o diâmetro médio ponderado dos agregados (DMP), determinado por agitação em água dos agregados entre 4,76 e $8,00 \mathrm{~mm}$, conforme método de Kemper \& Chepil (1965).

Os atributos quími cos analisados foram carbono orgânico, $\mathrm{Al}, \mathrm{Ca}$, Mg e $\mathrm{K}$ trocáveis, $\mathrm{pH}$ em água e em solução de $\mathrm{KCl} 1 \mathrm{~mol} \mathrm{~L}^{-1}$, segundo métodos apresentados por Tedesco et al. (1995). O ponto de efeito salino nulo (PESN) foi determinado por titulação potenciométrica, em amostras compostas, obtidas a partir da mistura das quatro repetições, segundo EMBRAPA (1997). A partir dos valores de $\mathrm{pH}$ e PESN, calculou-se o potencial elétrico superficial ( $\Psi 0)$, expresso em $\mathrm{mV}$, segundo Raij \& Peech (1972), utilizando a equação simplificada de Nernst: $\Psi 0=59,1$ (PESN - pH).

Os resultados foram submetidos à análise da variância pel o teste $F$, com posterior comparação de médias pelo teste de Tukey, a $5 \%$. E fetuou-se análise de correlação de Pearson entre DMP e grau de floculação da argila, em relação ao demais atributos químicos e el etroquímicos do solo.

\section{RESULTADOS E DISCUSSÃO}

\section{Atributos químicos e eletroquímicos do solo}

A calagem mel horou o ambiente edáfico na camada analisada, ao el evar o pH do solo, os teores de Ca e Mg trocáveis, a soma de bases e a CTC. Houve redução significativa nos teores de $\mathrm{Al}^{3+}$ trocável, observada já na dose de $4,5 \mathrm{Mg} \mathrm{ha}^{-1}$ de cal cário, acarretando, assim, redução na saturação por alumínio. A aplicação de cal cário, por outrolado, não influiu nos teores de $K$ trocável e na condutividade elétrica do solo (Quadro 1).

A adição de adubos fosfatados durante cinco safras elevou linearmente os teores de fósforo disponível no solo, passando de $3,9 \mathrm{mg} \mathrm{kg}^{-1}$ de $\mathrm{P}$, quando da adição anual média de $42 \mathrm{~kg} \mathrm{ha}^{-1}$ de $_{2} \mathrm{O}_{5}$, para $11,5 \mathrm{mg} \mathrm{kg}^{-1}$ de $\mathrm{P}$, quando da aplicação anual média de $168 \mathrm{~kg} \mathrm{ha}^{-1}$ de $\mathrm{P}_{2} \mathrm{O}_{5}$, el evando, conseqüentemente, o rendimento das culturas (Ernani et al., 2000).

A correção da acidez do solo não influenciou os teores de carbono orgânico, diferindo do que foi constatado por Ernani et al. (2002) em solos semelhantes e por Azevedo et al. (1996), que estudaram a dinâmica evolutiva também de um Latossolo Bruno do Rio Grande do Sul submetido à calagem. Nestes casos, houve estímulo à decomposição mediante a calagem, atribuído ao aumento populacional dos microrganismos decorrente da el evação do pH do solo. 
Quadro 1. Efeito da calagem e adubação fosfatada sobre os atributos eletroquímicos de um Latossolo Bruno argiloso álico

\begin{tabular}{|c|c|c|c|c|c|c|c|c|c|c|c|c|c|c|}
\hline $\mathrm{P}_{2} \mathrm{O}_{5}{ }^{(1)}$ & CE & $\mathbf{p H}$ & $\Delta \mathrm{pH}$ & PES & PESN & Corg. & $\mathbf{P}$ & $\mathrm{Ca}$ & Mg & $\mathbf{K}$ & Al & SB & СтC & $\mathbf{m}$ \\
\hline $\mathrm{kg} \mathrm{ha}^{-1}$ & $\mathrm{mS} \mathrm{m}^{-1}$ & & & $\mathrm{mV}$ & & $\mathrm{g} \mathrm{kg}^{-1}$ & $\mathrm{mg} \mathrm{kg}^{-1}$ & 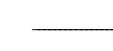 & - & $-\mathrm{cmol}_{c}$ & $\mathrm{~kg}^{-1}$ & & & $\%$ \\
\hline \multicolumn{15}{|c|}{ Sem cal cário } \\
\hline $\begin{array}{l}P_{42} \\
P_{84} \\
P_{126} \\
P_{168}\end{array}$ & $\begin{array}{l}22 \\
20 \\
20 \\
22\end{array}$ & $\begin{array}{l}4,6 \\
4,7 \\
4,7 \\
4,7\end{array}$ & $\begin{array}{l}-0,8 \\
-0,9 \\
-0,9 \\
-0,9\end{array}$ & $\begin{array}{l}-48 \\
-51 \\
-53 \\
-53\end{array}$ & $\begin{array}{l}3,8 \\
3,8 \\
3,8 \\
3,8\end{array}$ & $\begin{array}{l}27,5 \\
26,6 \\
26,8 \\
27,1\end{array}$ & $\begin{array}{r}5,4 \\
6,8 \\
7,4 \\
12,3\end{array}$ & $\begin{array}{l}0,9 \\
1,2 \\
1,5 \\
1,6\end{array}$ & $\begin{array}{l}0,5 \\
0,7 \\
0,8 \\
0,8\end{array}$ & $\begin{array}{l}0,5 \\
0,4 \\
0,4 \\
0,4\end{array}$ & $\begin{array}{l}3,9 \\
3,7 \\
3,4 \\
3,4\end{array}$ & $\begin{array}{l}1,9 \\
2,3 \\
2,8 \\
2,8\end{array}$ & $\begin{array}{l}5,7 \\
6,0 \\
6,2 \\
6,2\end{array}$ & $\begin{array}{l}67 \mathrm{~A} \\
61 \mathrm{~A} \\
56 \mathrm{~A} \\
54 \mathrm{~A}\end{array}$ \\
\hline Média & 21 & $4,7 \mathrm{C}$ & $-0,9 \mathrm{~A}$ & $-51 A$ & 3,8 & 27,0 & $8,0 \mathrm{~A}$ & $1,3 \mathrm{C}$ & $0,7 \mathrm{C}$ & 0,4 & $3,6 \mathrm{~A}$ & $2,4 \mathrm{C}$ & $6,0 \mathrm{C}$ & $60 \mathrm{~A}$ \\
\hline \multicolumn{15}{|c|}{ 4,5 $\mathrm{Mg} \mathrm{ha}^{-1}$ calcário } \\
\hline $\begin{array}{l}P_{42} \\
P_{84} \\
P_{126} \\
P_{168}\end{array}$ & $\begin{array}{l}30 \\
23 \\
26 \\
25\end{array}$ & $\begin{array}{l}5,5 \\
5,5 \\
5,6 \\
5,6\end{array}$ & $\begin{array}{l}-1,0 \\
-1,1 \\
-1,1 \\
-1,2\end{array}$ & $\begin{array}{l}-101 \\
-80 \\
-86 \\
-78\end{array}$ & $\begin{array}{l}3,8 \\
4,1 \\
4,1 \\
4,3\end{array}$ & $\begin{array}{l}26,7 \\
25,6 \\
26,0 \\
25,4\end{array}$ & $\begin{array}{r}3,1 \\
5,9 \\
7,2 \\
11,1\end{array}$ & $\begin{array}{l}4,3 \\
4,4 \\
4,7 \\
5,0\end{array}$ & $\begin{array}{l}3,8 \\
3,1 \\
4,0 \\
4,2\end{array}$ & $\begin{array}{l}0,5 \\
0,4 \\
0,5 \\
0,4\end{array}$ & $\begin{array}{l}0,5 \\
0,5 \\
0,4 \\
0,4\end{array}$ & $\begin{array}{l}8,5 \\
7,9 \\
9,2 \\
9,6\end{array}$ & $\begin{array}{r}9,0 \\
8,5 \\
9,6 \\
10,0\end{array}$ & $\begin{array}{l}6 \text { B } \\
6 \text { B } \\
4 \text { B } \\
4 \text { B }\end{array}$ \\
\hline Média & 26 & $5,5 \mathrm{~B}$ & $-1,1 \mathrm{~B}$ & $-87 B$ & 4,1 & 25,9 & $6,8 \mathrm{AB}$ & $4,6 \mathrm{~B}$ & $3,8 \mathrm{~B}$ & 0,4 & $0,5 \mathrm{~B}$ & $8,8 \mathrm{~B}$ & $9,3 \mathrm{~B}$ & $5 \mathrm{~B}$ \\
\hline \multicolumn{15}{|c|}{$9,0 \mathrm{Mg} \mathrm{ha}^{-1}$ calcário } \\
\hline $\begin{array}{l}P_{42} \\
P_{84} \\
P_{126} \\
P_{168}\end{array}$ & $\begin{array}{l}27 \\
32 \\
28 \\
26\end{array}$ & $\begin{array}{l}6,4 \\
6,3 \\
6,2 \\
6,3\end{array}$ & $\begin{array}{l}-1,1 \\
-1,1 \\
-1,0 \\
-1,1\end{array}$ & $\begin{array}{l}-115 \\
-117 \\
-112 \\
-116\end{array}$ & $\begin{array}{l}4,4 \\
4,3 \\
4,3 \\
4,3\end{array}$ & $\begin{array}{l}23,9 \\
24,0 \\
26,3 \\
24,8\end{array}$ & $\begin{array}{r}3,2 \\
4,3 \\
7,7 \\
11,2\end{array}$ & $\begin{array}{l}7,0 \\
7,0 \\
6,7 \\
7,0\end{array}$ & $\begin{array}{l}7,0 \\
6,7 \\
6,3 \\
6,7\end{array}$ & $\begin{array}{l}0,4 \\
0,4 \\
0,4 \\
0,4\end{array}$ & $\begin{array}{l}0,1 \\
0,2 \\
0,2 \\
0,1\end{array}$ & $\begin{array}{l}14,4 \\
14,1 \\
13,4 \\
14,1\end{array}$ & $\begin{array}{l}14,5 \\
14,3 \\
13,6 \\
14,2\end{array}$ & $\begin{array}{ll}1 & \mathrm{~B} \\
1 & \mathrm{~B} \\
1 & \mathrm{~B} \\
1 & \mathrm{~B}\end{array}$ \\
\hline Média & 28 & $6,3 \mathrm{~A}$ & $-1,1 \mathrm{~B}$ & $-115 C$ & 4,3 & 24,8 & $6,6 \mathrm{~B}$ & $6,9 \mathrm{~A}$ & $6,7 \mathrm{~A}$ & 0,4 & $0,1 \mathrm{~B}$ & $14,0 \mathrm{~A}$ & $14,2 \mathrm{~A}$ & $1 \mathrm{~B}$ \\
\hline \multicolumn{15}{|c|}{ E feito médio do fósforo } \\
\hline $\begin{array}{l}P_{42} \\
P_{84} \\
P_{126} \\
P_{168}\end{array}$ & $\begin{array}{l}26 \\
25 \\
25 \\
24\end{array}$ & $\begin{array}{l}5,5 \\
5,5 \\
5,5 \\
5,5\end{array}$ & $\begin{array}{l}-1,0 \\
-1,0 \\
-1,0 \\
-1,1\end{array}$ & $\begin{array}{l}-88 \\
-83 \\
-84 \\
-83\end{array}$ & $\begin{array}{l}4,0 \\
4,1 \\
4,1 \\
4,1\end{array}$ & $\begin{array}{l}26,1 \\
25,4 \\
26,4 \\
25,8\end{array}$ & $\begin{array}{c}3,9 \mathrm{c} \\
5,6 \mathrm{bc} \\
7,4 \mathrm{~b} \\
11,5 \mathrm{a}\end{array}$ & $\begin{array}{l}4,0 \mathrm{~b} \\
4,2 \mathrm{ab} \\
4,3 \mathrm{ab} \\
4,6 \mathrm{a}\end{array}$ & $\begin{array}{l}3,8 \\
3,5 \\
3,7 \\
3,9\end{array}$ & $\begin{array}{l}0,5 a \\
0,4 a b \\
0,4 a b \\
0,4 b\end{array}$ & $\begin{array}{l}1,5 \\
1,5 \\
1,3 \\
1,3\end{array}$ & $\begin{array}{l}8,3 \\
8,1 \\
8,5 \\
8,9\end{array}$ & $\begin{array}{r}9,8 \\
9,6 \\
9,8 \\
10,1\end{array}$ & $\begin{array}{l}25 a \\
23 a b \\
20 a b \\
20 b\end{array}$ \\
\hline \multicolumn{15}{|c|}{ Análise de variância, $\mathrm{P}>\mathrm{F}$} \\
\hline Cal cário & ns & $<0,001$ & $<0,001$ & $<0,001$ & -- & ns & 0,033 & $<0,001$ & $<0,001$ & ns & $<0,001$ & $<0,001$ & $<0,001$ & $<0,001$ \\
\hline Fósforo & ns & ns & ns & ns & -- & ns & $<0,001$ & 0,029 & ns & 0,051 & ns & ns & ns & 0,019 \\
\hline I nteração & ns & ns & ns & ns & -- & ns & ns & ns & ns & ns & ns & ns & ns & 0,048 \\
\hline
\end{tabular}

(1) Níveis de fósforo: $\mathrm{P}_{42}, \mathrm{P}_{84}, \mathrm{P}_{126}$ e $\mathrm{P}_{168}$, correspondendo, respectivamente, a aplicações anuais de $60,120,180$ e $240 \mathrm{~kg}$ ha-1 de $\mathrm{P}_{2} \mathrm{O}_{5}$ no primeiro ano; 40, 80, 120 e $160 \mathrm{~kg} \mathrm{ha}^{-1} \mathrm{de}_{2} \mathrm{O}_{5}$, no segundo, quarto e quinto ano, e 30, 60, 90 e $120 \mathrm{~kg} \mathrm{ha}^{-1}$ de $\mathrm{P}_{2} \mathrm{O}_{5}$, no terceiro ano. $\mathrm{CE}=$ condutividade elétrica; PES = potencial elétrico superficial; ns = não-significativo a $5 \%$; - = não analisado.

A calagem aumentou o ponto de efeito salino nulo (PESN) do solo de 3,8, no solo sem calagem, para 4,3 quando foram adicionados $9,0 \mathrm{Mg} \mathrm{ha}^{-1} \mathrm{de}$ calcário. Estevalor foi próximo a 4,6, referido como PESN da caulinita, a qual é o mineral de argila predominante nesse solo. Em todas as doses de calcário, o PESN foi inferior ao pH em água desses solos, evidenciando o predomínio de carga líquida negativa nesses colói des, o quetambémé confirmado pelos valores negativos de $\Delta \mathrm{pH}$ (Quadro 1). O aumento do PESN do solo ocasionado pela calagem pode ser atribuído à adsorção específica de Ca e Mg por complexos de esfera interna, que levam à formação de cargas positivas nas superfícies dos colóides (Sposito, 1989). Outro fator que pode ter contribuído para elevar o PESN é a tendência de redução nos teores de carbono orgânico, resultante da calagem, uma vez que essas duas variáveis são inversamente relacionadas (Siqueira et al., 1990).
A aplicação de cal cário el evou o potencial elétrico superficial negativo, que passou de $-51 \mathrm{mV}$, no tratamento sem cal cário, para $-115 \mathrm{mV}$, na dose de 9,0 $\mathrm{Mg} \mathrm{ha}^{-1}$ de calcário (Quadro 1), graças ao aumento das cargas el étricas negativas decorrentes da adsorção química de hidroxilas na superfície dos óxidos deFeeAl enas arestas quebradas da caulinita (McBride, 1989), bem como da ionização de grupos funcionais da matéria orgânica, principalmente os carboxílicos (Uehara \& Gillmann, 1981).

A adubação fosfatada não al terou oPESN do solo, nem o potencial elétrico superficial, possivelmente porqueas doses deP empregadas foram relativamente baixas, tratando-se de um solo argiloso e com alto tamponamento. Entretanto, relatos na literatura indicam que a adição de fósforo diminui oPESN (Wann \& Uehara, 1978; Dolui et al., 1991). Além disso, a adsorção deP, através de compl exos de esfera interna em óxidos e hidróxidos de Fe e Al, age de forma 
semel hanteà cal agem, aumentandoas cargas negativas na superfície das partículas, o que incrementaria o potencial elétrico negativo superficial, como verificado por Sawhney (1974) e por Lima et al. (2000), em solos altamente intemperizados de Minas Gerais.

Em outro estudo, realizado duranteseis anos por Dynia \& Camargo (1998), num Latossolo do estado de Goiás, observou-se que tanto a adição de fosfatos quanto a combinação cal agem e fósforo ocasionaram aumento na carga elétrica líquida e na CTC efetiva, no $\mathrm{pH}$ original do solo, mas não alteraram o PESN do solo. Essas alterações químicas podem influenciar a floculação e a dispersão das partículas, que dependem da interação el etrostática entre el as e da habilidade do ânion fosfato em reduzir a ação floculante dos cátions (EI Swaify, 1973).

\section{Modificações na estrutura do solo}

A calagem diminuiu o grau defloculação da argila de $69 \%$, em média, no solo não calcariado, para aproximadamente $58 \%$, quando se adicionaram 9,0 $\mathrm{Mg} \mathrm{ha}^{-1}$ de calcário (F igura 1a). Esta redução está, provavel mente, relacionada com o aumento da espessura da dupla camada elétrica difusa dos colóides atribuído à criação de cargas negativas, a qual se evidencia pelo aumento na CTC e pela substituição do $\mathrm{Al}^{3+}$ pelo $\mathrm{Ca}^{2+}{\mathrm{e} \mathrm{Mg}^{2+} \text { no complexo }}^{2}$ de troca. Outra modificação resultante da calagem com influência sobre os atributos físicos do sol o é a diminuição na atividade de $\mathrm{Al}^{3+} \mathrm{e} \mathrm{H}^{+}$na solução do solo, que são os principais agentes floculantes em solos ácidos (Morelli \& Ferreira, 1987). Nesse caso, há precipitação de polímeros de hidróxidos de Al, pelo aumento do $\mathrm{pH}$ do solo.

Todas essas alterações, ocorridas na superfície das partículas, refletem a diminuição das forças de atração entre os col ói des, facilitando a dispersão em meioaquoso. Essa dispersão dos colóides podereduzir os fluxos de solução e ar do solo, pois diminui o diâmetro e o volume dos poros (Roth \& Pavan, 1991). Estes autores observaram que a correção da acidez com cal cário, num Latossolo do Paraná, aumentou a argila dispersa em água com conseqüenter edução da taxa de infiltração de água no solo.

Houve correlação positiva significativa entre o grau de floculação da argila com Al trocável, saturação por Al ecarbono orgânico do sol o e negativa com $\mathrm{pH}$ em água e em $\mathrm{KCl}, \mathrm{CTC}, \mathrm{SB}, \mathrm{Ca}, \mathrm{Mg}$ e potencial elétrico superficial negativo (Quadro 2).

A adição de fosfato ao solo, diferentemente da calagem, não interferiu sobre o grau de floculação da argila durante o período avaliado (Figura 1a). Esse comportamento refletiu-se também na ausência de correlação entre o grau de floculação e os teores de $P$ no solo (Quadro 2). Por outro lado, al guns estudos com solos de regiões tropicais têm evidenciado efeito negativo da adição de fósforo sobre atributos físicos de sol os ou horizontes com baixos teores de carbono orgânico.
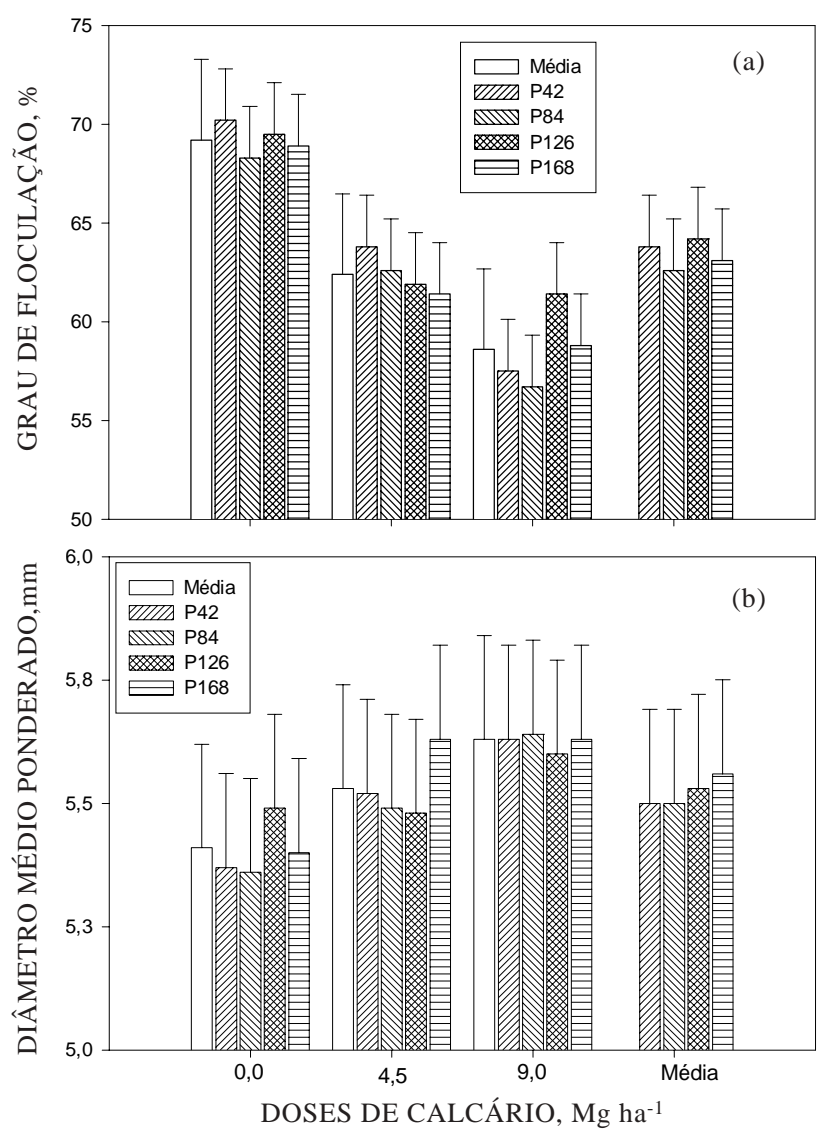

Figura 1. Efeito da calagem e adubação fosfatada sobre o grau de floculação da argila (a) e sobre o diâmetro médio ponderado dos agregados (b) de um Latossolo Bruno argiloso álico. Barras verticais entre médias do fator fósforo e entre médias do fator calcário correspondem às diferenças mínimas significativas calculadas pelo teste de Tukey, a $5 \%$.

Silva et al. (1999) verificaram, em dois solos do estado de Minas Gerais, com 12 e $22 \mathrm{~g} \mathrm{~kg}^{-1}$ de C orgânico, uma dispersão de argila coma aplicação defosfato, tornando-os mais sujeitos à compactação. A aplicação de $P$ nesses solos alterou também os limites de consistência, reduzindo a amplitude da zona de friabilidade e a capacidade de trabal ho e tráfego dos solos. O aumento na dispersão das argilas pela adição de fósforo foi também relatado por Lima et al. (2000) em solos altamente intemperizados de Minas Gerais. A adsorção de fósforo aumenta as cargas negativas na superfície das partículas e favorece a dispersão dos colói des, o que foi mais evidente no horizonte $B$ em relação ao $A$, graças à menor influência da matéria orgânica como agregante em subsuperfície. Observou-se também que, quanto maior a diferença entre o ponto isoelétrico e o pH do solo, maiores as quantidades de argila dispersa. 


\section{Quadro 2. Coeficientes de correlação de Pearson entre indicadores da estabilidade da estrutura (grau de floculação, \%, e diâmetro médio ponderado, $\mathrm{mm}$ ) e atributos químicos e eletroquímicos de um Latossolo Bruno argiloso álico}

\begin{tabular}{|c|c|c|}
\hline Atributo do solo & Grau de floculação & Diâmetro médio ponderado \\
\hline & $\%$ & $\mathrm{~mm}$ \\
\hline Condutividade elétrica ( $\mathrm{mS} \mathrm{m}^{-1}$ ) & $-0,39 * *$ & $0,14^{\text {ns }}$ \\
\hline $\mathrm{pH}$ em água & $-0,89 * *$ & $0,48^{* *}$ \\
\hline $\mathrm{pH}$ em KCl M & $-0,86 * *$ & $0,43 * *$ \\
\hline Potencial elétrico superficial negativo (mV) & $-0,85^{* *}$ & $0,46 * *$ \\
\hline Carbono orgânico $\left(\mathrm{g} \mathrm{kg}^{-1}\right)$ & $0,54 * *$ & $-0,48 * *$ \\
\hline$P\left(\mathrm{mg} \mathrm{kg}^{-1}\right)$ & $0,15^{\text {ns }}$ & $0,01^{\text {ns }}$ \\
\hline $\mathrm{Ca}\left(\mathrm{cmol}_{\mathrm{c}} \mathrm{kg}^{-1}\right)$ & $-0,89 * *$ & $0,45^{* *}$ \\
\hline $\mathrm{Mg}\left(\mathrm{cmol}_{\mathrm{c}} \mathrm{kg}^{-1}\right)$ & $-0,89 * *$ & $0,48 * *$ \\
\hline $\mathrm{K}\left(\mathrm{cmol}_{\mathrm{c} \mathrm{kg}} \mathrm{kg}^{-1}\right)$ & $0,02^{\text {ns }}$ & $-0,23^{n s}$ \\
\hline $\mathrm{Al}\left(\mathrm{cmol}_{\mathrm{c}} \mathrm{kg}^{-1}\right)$ & $0,81 * *$ & $-0,38 * *$ \\
\hline Soma de bases (SB) $\left(\mathrm{cmol}_{\mathrm{c}} \mathrm{kg}^{-1}\right)$ & $-0,90 * *$ & $0,47 * *$ \\
\hline CTC $\left(\mathrm{cmol}_{\mathrm{c}} \mathrm{kg}^{-1}\right)$ & $-0,88 * *$ & $0,48 * *$ \\
\hline Saturação por alumínio (\%) & $0,81 * *$ & $-0,38 * *$ \\
\hline Grau de floculação & - & $-0,48 * *$ \\
\hline
\end{tabular}

** $\mathrm{e}^{\text {ns }}=$ Significativo a 1 \% e não-significativo.

O diâmetro médio ponderado dos agregados (DMP), que é um indicativo da estabilidade da estrutura do solo, não foi afetado pela calagem e adubaçãofosfatada (Figura 1b), apesar das alterações químicas e el etroquímicas do solo com reflexos negativos na floculação de argila no solo pela calagem. Ao contrário, verificou-seligeiro aumento no DMP pela aplicação de calcário. De maneira geral, esses solos tamponados do planalto sulbrasileiro, com teores satisfatórios de carbono orgânico, mostram alta estabilidade da estrutura, mantendo os agregados estáveis.

Nas condições estudadas, haveria um equilíbrio em termos de estabilidade da estrutura, sem interferência significativa das práticas adotadas. Diversos fatores estariam ligados a este equilíbrio, sendo a sua magnitude dependente do tipo de solo, das condições climáticas e do tempo de avaliação considerado (Castro Filho \& Logan, 1991). Por um lado, verificou-se aumento da dispersão dos colói des e tendência de aumento da oxidação do carbono orgânico, que favoreceriam a desagregação. Contrariamente, a aplicação de cal cário promoveu, em alguns casos, melhoria física, como num Latossolo Roxo, aumentando a estabilidade de agregados e a infiltração de água no solo (Roth et al., 1986). Essa mel horia estaria ligada ao estímulo ao aumento da população mi crobiana, que intensifica a decomposição da matéria orgânica e libera inúmer os compostos que atuam na ligação entre as partículas do solo. Outro mecanismo pelo qual a calagem interfere favoravel mente na agregação do solo é pela participação do $\mathrm{Ca}^{2+}$ na formação de complexos argila-húmus (Muneer \& Oades, 1989), bem como pel o maior desenvolvimento das culturas num ambiente edáfico mais adequado do ponto de vista químico.

Butierres (1980), avaliando as alterações nos atributos físicos do solo, advindas da adição de cal cário e fosfatos em laboratório, observou que a aplicação de cal cário di minuiu o grau defloculação das argilas de um L atossol o Roxo distrófico, mas aumentou no caso de um Planossolo. Para a aplicação de fósforo, verificou-se ligeira diminuição deste atributo nos dois solos. Em outro experimento semelhante, Morelli \& Ferreira (1987) verificaram quea calagem e a adição de fósforo diminuíram a estabilidade da estrutura eotamanho dos agregados de um Latossolo Roxo do Rio Grande do Sul. Este efeito esteve relacionado com o aumento na dispersão dos colóides, provocando, assim, redução na percolação de água no solo, possivelmente ligada à obstrução dos poros por partículas finas dispersas. Esses efeitos foram mais evidentes no horizonte $B$ em relação ao horizonte $A$, em virtude do efeito cimentante da matéria orgânica do solo, a qual é mais abundante na camada superficial.

Essa diferenciação na estabilidade estrutural do solo provocada pela matéria orgânica já foi comprovada em ensaios de laboratório, que demonstram a influência do tipo de material orgânico e, em especial, das substâncias húmicas sobre a estabilidade coloidal de suspensões com argilas. O aumento da floculação das partículas estaria ligado a modi ficações el etrostáticas derivadas dos compostos orgânicos (K retzschmar et al., 1997). No campo, o papel da matéria orgânica é sal ientado por Westerhof et al. (1999), que, trabalhando com Latossol os da região de Planaltina, DF, verificaram 
diminuição na estabilidade dos agregados pela calagem, principalmente em solos revolvidos mecanicamente e com baixo teor de carbono orgânico. No sol osem preparo, esse efeito prejudicial da adição de calcário sobre a agregação não foi observado, o que possivel mente se deveu aos maiores teores de carbono orgânico nesta situação.

O DMP mostrou-se positivamentecorrelacionado com os teores de $\mathrm{Ca}, \mathrm{Mg}, \mathrm{CTC}$, SB e com o pH em água e em $\mathrm{KCl}$ desse solo. Esse atributo teve correlação negativa com o carbono orgânico, Al, potencial el étrico superficial egrau de floculação da argila (Quadro 2). Os coeficientes de correl ação, em todos esses casos, mostraram-se significativos, mas foram baixos, entre 0,4 e 0,5, indicando que a agregação do sol o depende da interação de uma série defatores do solo, além daquel es de influência direta da calagem e adição de adubos, dependendo do tipo de solo e mineralogia, teor de nutrientes, Fe e Al, atividade biológica, umedecimento e secagem e manejo do solo (Seta \& Karathanasis, 1997).

\section{CONCLUSÕES}

1. A correção da acidez do sol o el evou o PE SN e o potencial elétrico superficial negativo, pelo aumento da carga líquida negativa das partículas.

2. A calagem não interferiu no diâmetro médio dos agregados, provavelmente em razão do efeito benéfico da matéria orgânica sobre os atri butos físicomecâni cos do sol o eda açãoindireta da calagem sobre a agregação, pelo estímulo à atividade biológica do solo.

3. A calagem nesse sol o com al to tamponamento interferiu negativamente na floculação das partículas, mas não comprometeu a estabilidade estrutural do solo.

\section{LITE RATURA CITADA}

ALBUQUERQUE, J.A.; BAYER, C.; ERNANI, P.R. \& FONTANA, E.C. Propriedades físicas e el etroquímicas de um Latossolo Bruno afetadas pela calagem. R. Bras. Ci. Solo, 24:295-300, 2000.

AZEVEDO, A.C.; KAMPF, N. \& BOHNEN, H. Alterações na dinâmica evolutiva de Latossolo Bruno pela calagem. R. Bras. Ci. Solo, 20:191-198, 1996.

BUTIERRES, M.F.M. Efeito do cal cário e fosfato de potássio no ponto de zero carga (PZC) e grau de floculação de três sol os do Rio Grande do Sul. Santa Maria, Universidade Federal do Rio Grande do Sul, 1980. 59p. (Tese de Mestrado)

CASTRO FILHO, C. \& LOGAN, T.J. Liming effects on the stability and erodibility of some Brazilian Oxisols. Soil Sci. Soc. Am. J ., 55:1407-1413, 1991.
DOLUI, A.K.; MUKHOPADHYAY, M. \& NAYEK, A.K. Effect of phosphatic fertilizer on charge characteristics of soils in the tropics. Int. J . Trop. Agric., 9:259-266, 1991.

DYNIA, J .F. \& CAMARGO, O.A. Effects of liming, green manuring, and phosphate addition on electrochemical attributes of an Oxisol from central Brazil. Comm. Soil Sci. Plant Anal., 29:755-762, 1998.

EL SWAIFY, S.A. Structural changes in tropical soils due to anions in irrigation water. Soil Sci., 115:64-72, 1973.

EMPRESA BRASILEIRA DE PESQUISA AGROPECUÁRIA EMBRAPA. CentroNacional de Pesquisa de Solos. Manual de métodos de análise de solo. 2.ed. Rio de J aneiro, 1997. $212 p$.

ERNANI, P.R.; BAYER, C. \& MAESTRI, L. Corn yield as affected by liming and tillage system on an acid Brazilian oxisol. Agron. J ., 94:305-309, 2002.

ERNANI, P.R.; NASCIMENTO, J .A.L.; CAMPOS, M.L. \& CAMILLO, R.J. Influência da combinação de fósforo e cal cário no rendimento de milho. R. Bras. Ci. Solo, 24:537544, 2000.

FONTES, M.P.F.; GJ ORUP, G.B.; ALVARENGA, R.C. \& NASCIF, P.G.S. Calcium salts and mechanical stress effects on water-dispersible clay of Oxisols. Soil Sci. Soc. Am. J ., 59:224-227, 1995.

HAYNES, R.J . Lime and phosphate in the soil-plant system. Adv. Agron., 37:249-315, 1984.

HAYNES, R.J . \& NAIDU, R. Influence of lime, fertilizer and manure applications on soil organic matter content and soil physical conditions: A review. Nutr. Cycling Agroecosy., 51:123-137, 1998.

KEMPER, W.D. \& CHEPIL, W.S. Sizedistribution of aggregates. In: BLACK, C.A.; EVANS, D.D.; WHITE, J.L.; ENSMINGER, L.E. \& CLARCK, F.E., eds. Methods of soil analysis. Madison, American Society of Agronomy, 1965. p.499-510. (Agronomy Monograph, 9)

KRETZSCHMAR, R.; HESTERBERG, D. \& STICHER, H. Effects of adsorbed humic acid on surface charge and flocculation of kaolinite. Soil Sci. Soc. Am. J ., 61:101-108, 1997.

LIMA, J .M.; ANDERSON, S.J . \& CURI, N. Phosphate-induced clay dispersion as related to aggregate size and composition in Hapludoxs. Soil Sci. Soc. Am. J ., 64:892-897, 2000.

MCBRIDE, M.B. Surface chemistry of soil minerals. In: DIXON, J .B. \& WEED, S.B., eds. Minerals in soil environments. 2.ed. Madison, Soil Science Society of America, 1989. p.3588.

MORELLI, M. \& FERREIRA, E.B. Efeito do carbonato de cálcio e do fosfato diamônico em propriedades eletroquímicas e físicas de um Latossolo. R. Bras. Ci. Solo, 11:1-6, 1987.

MUNEER, M. \& OADES, J.M. The role of Ca-organic interactions in soil aggregatestability. III. Mechanisms and models. Aust. J . Soil Res., 27:411-423, 1989.

RAIJ , B. van \& PEECH, M. Electrochemical properties of some Oxisols and Alfisols of the tropics. Soil Sci. Soc. Am. Proc., 36:587-593, 1972. 
ROTH, C.H. \& PAVAN, M.A. Effect of lime and gypsum on clay dispersion and infiltration in samples of a Brazilian Oxisol. Geoderma, 48:351-361, 1991.

ROTH, C.H.; PAVAN, M.A.; CHAVES, J.C.D.; MEYER, B. \& FREDE, H.G. Efeitos da aplicação de cal cário e gesso sobre a estabilidade de agregados e infiltração de água em um Latossolo Roxo cultivado com cafeeiros. R. Bras. Ci. Solo, 10:163-166, 1986.

SANTA CATARINA. Gabinete de Planejamento e Coordenação Geral. Sub-chefia de Estatística, Geografia e I nformática. Atlas de Santa Catarina. Rio deJ aneiro, Aerofoto Cruzeiro, 1986. 176p.

SAWHNEY, B.L. Charge characteristics of soils as affected by phosphatesortion. Soil Sci. Soc. Am. Proc., 38:159-160, 1974.

SETA, A.K. \& KARATHANASIS, A.D. Water dispersible colloids and factors influencing their dispersibility from soil aggregates. Geoderma, 74:255-266, 1997.

SIQUEIRA, C.; LEAL, J .R. \& VELLOSO, A.C.X. Eletroquímica de solos tropicais de carga variável. II. Quantificação do efeito da matéria orgânica sobre o ponto de carga zero. R. Bras. Ci. Solo, 14:13-17, 1990.
SILVA, R.B.; LIMA, J.M. \& DIAS JÚNIOR, M.S. Efeito da adsorção de fosfato em parâmetros físicos e na compressibilidade de solos tropicais. R. Bras. Ci. Solo, 23:219-226, 1999.

SPOSITO, G. The chemistry of soils. New York, Oxford University Press, 1989. 277p.

TEDESCO, M.J .; GIANELLO, C.; BISSANI, C.A. BOHNEN, H. \& VOLKWEISS, S. Análise de solo, plantas e outros materiais. Porto Alegre, Universidade Federal do Rio Grande do Sul, 1995. 174p.

UEHARA, G. \& GILLMAN, G. The mineralogy, chemistry and physics of tropical soils with variable chargeclays. Boulder, Westview Press, 1981. 170p.

WANN, S.S. \& UEHARA, G. Surface charge manipulation of constant surface potential soil colloids. I. Relation to sorbed phosphorus. Soil Sci. Soc. Am. J ., 42:565-570, 1978.

WESTERHOF, R.; BUURMAN, P.; van GRIETHUYSEN, C.; AYARZA, M.; VILELA, L. \& ZECH, W. Aggregation studied by laser diffraction in relation to plowing and liming in the Cerrado region in Brazil. Geoderma, 90:277-290, 1999. 